\title{
Review
}

\section{From migration to settlement: the pathways, migration modes and dynamics of neurons in the developing brain}

\author{
By Yumiko HatanakA, ${ }^{* 1}$ Yan Zhu, ${ }^{* 2}$ Makio ToRIgoe, ${ }^{* 3}$ \\ Yoshiaki KITA $^{* 4}$ and Fujio MuRAKAMI*5,† \\ (Communicated by Shigekazu NAGATA, M.J.A.)
}

\begin{abstract}
Neuronal migration is crucial for the construction of the nervous system. To reach their correct destination, migrating neurons choose pathways using physical substrates and chemical cues of either diffusible or non-diffusible nature. Migrating neurons extend a leading and a trailing process. The leading process, which extends in the direction of migration, determines navigation, in particular when a neuron changes its direction of migration. While most neurons simply migrate radially, certain neurons switch their mode of migration between radial and tangential, with the latter allowing migration to destinations far from the neurons' site of generation. Consequently, neurons with distinct origins are intermingled, which results in intricate neuronal architectures and connectivities and provides an important basis for higher brain function. The trailing process, in contrast, contributes to the late stage of development by turning into the axon, thus contributing to the formation of neuronal circuits.
\end{abstract}

Keywords: neuronal migration, cortical interneuron, precerebellar neuron, tangential migration, radial migration, non-directed migration

\section{Introduction}

The central nervous system (CNS) is not a uniform structure, but is highlighted by two types of neuronal architectures: laminated structures and aggregates of neurons, called layers and nuclei, respectively. Neurons comprising specific layers or nuclei send axonal projections to specific targets and receive inputs from specific neuronal structures, thereby serving as functional units. Certain types of

*1 Division of Cerebral Circuitry, National Institute for Physiological Sciences, Okazaki, Aichi, Japan.

*2 Division of Brain Function, National Institute of Genetics, Mishima, Shizuoka, Japan.

*3 Lab Dev Gene Regulation, RIKEN, BSI, Wako, Saitama, Japan.

*4 Lab Mol Mech Thalamus Dev, RIKEN BSI, Wako, Saitama, Japan.

*5 Graduate School of Frontier Biosciences, Osaka University, Suita, Osaka, Japan.

$\dagger$ Correspondence should be addressed: F. Murakami, Graduate School of Frontier Biosciences, Osaka University, Suita, Osaka 560-8531, Japan (e-mail: murakami@fbs.osaka-u.ac.jp).

Note: The first two authors contributed equally to this work.

doi: $10.2183 /$ pjab.92.1

C2016 The Japan Academy neurons in the CNS form a third type of architecture by dispersing over a wide area that is also important for function. The establishment of these three neuronal architectures is an outcome of correct regulation and termination of neuronal migration.

Neuronal migration has been classified into radial migration and tangential migration. Growing evidence indicates that migratory processes are highly dynamic, with neurons changing their direction of migration and sometimes even switching modes between radial and tangential migration. Precise descriptions of the dynamics of neuronal migration and interrogation of the underlying control mechanisms are important for complete understanding of how neurons born out of progenitor zones manage to precisely reach their final destinations where they participate in neuronal circuit formation.

In this review we will describe the dynamic processes of neuronal migration and discuss current understanding of the underlying mechanisms by using mainly inhibitory cortical interneurons and neurons in the hindbrain as examples. 


\section{Modes of migration}

The migration of neurons has been traditionally categorized into two types, radial and tangential, depending on the axis of the migratory direction in relation to the pial surface of the neural tube. These migration types show dynamic behavior, as radial migration can occur in two opposing directions while tangential migration can occur in a directed or nondirected manner, and switching between the two types of migration may take place.

1) Radial migration. a) Pia-directed radial migration. The most well studied mode of migration is pia-directed radial migration (Fig. 1). The progenitors of neurons or neuroepithelial cells have been historically called radial glia because of the radial orientation of their processes and expression of glial fibrillary acidic protein. ${ }^{1)}$ Most neurons in the CNS are, directly or indirectly, generated from the radial glia. ${ }^{2), 3)}$ Soon after birth, postmitotic neurons are generally thought to migrate radially from the ventricular zone (VZ) in the orientation of the radial glial processes by radial migration. ${ }^{4)}$ Most of these neurons then approach the pial surface and settle away from the VZ.

A typical and much studied example of piadirected radial migration is that of cortical excitatory neurons after their departure from the cortical VZ. It is now understood that their journey to the cortical plate is not a straightforward trajectory of piadirected radial migration. These neurons transiently assume a multipolar shape in the intermediate and subventricular zones (IZ/SVZ), frequently changing their direction of migration ${ }^{5)}$ before radially migrating towards the pial surface.

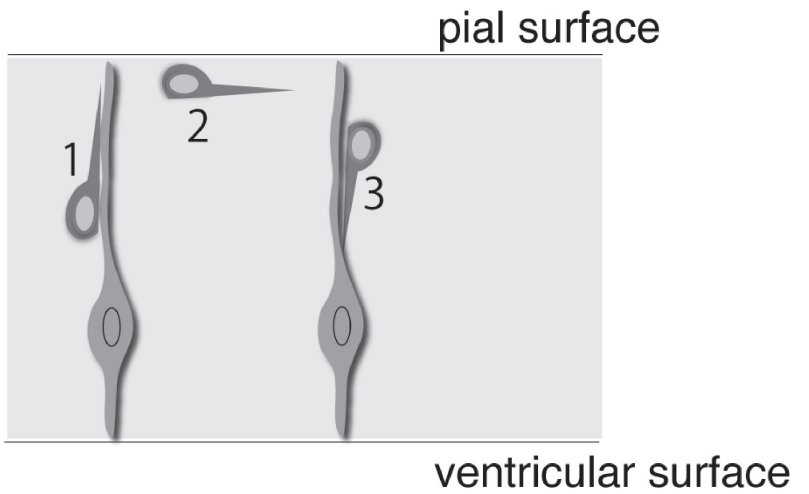

Fig. 1. Switching between migratory modes (radial and tangential). Some neurons, such as pontine neurons, initially migrate radially towards the pial surface (1), then migrate tangentially (2), and finally radially again away from the pia (3). b) Radial migration away from the pial surface. In contrast to pia-directed migration, certain neurons exhibit radial migration away from the pial surface. This type of radial migration is mostly performed by neurons that have executed tangential migration underneath the pial surface and includes granule cells of the cerebellum ${ }^{6)}$ and neurons of the pontine nuclei $(\mathrm{PN})$ in the hindbrain. ${ }^{7)}$ After leaving their site of generation, these neurons migrate circumferentially beneath the pial surface and then leave there by initiating radial migration away from the pia (Fig. 1).

2) Tangential migration. a) Directed tangential migration. Several types of neurons migrate tangentially. These include cortical interneurons in the IZ/SVZ, ${ }^{8)}$ cerebellar granule cells in the external granule layer ${ }^{9)}$ and precerebellar neurons such as PN neurons ${ }^{10)}$ neurons of the lateral reticular nucleus $(\mathrm{LRN})^{11)}$ and facial motor neurons in the hindbrain. ${ }^{12)}$ These neurons begin to migrate tangentially soon after departing from their site of generation. They migrate toward specific directions, sometimes traversing across brain compartments such as rhombomeres (PN neurons and facial branchiomotor neurons) or pallium-subpallium boundaries (cortical interneurons).

b) Non-directed tangential migration. Conventionally, neurons have been thought to migrate in specific directions either radially or tangentially. However, accumulating evidence suggests that certain types of neurons exhibit migration in all directions, usually on the tangential plane. This phenomenon could be viewed as a sort of tangential migration, but the behavior of these neurons is quite distinct from those that migrate in specific directions. Typical examples of neurons that conduct nondirected tangential migration are cortical interneurons in the marginal zone (MZ). These neurons migrate in all directions in the MZ beneath the pial surface. Moreover, these neurons repeatedly change their direction in a manner described as a random walk. ${ }^{13)}$

3) Dynamics of neuronal migration. a) Switching migration modes. Radial and tangential migrations are not isolated events, as several types of neurons switch their migration mode between tangential and radial and between directed and nondirected. The result of switching is a complete change in the direction of migration.

Most tangentially migrating neurons may have first migrated radially from the VZ towards the pial surface, and some may thereafter switch back to radial migration. For example, a time-lapse analysis 
of tangentially migrating granule cells in the external granular layer of the cerebellum showed that a descending process into the molecular layer appears from the cell body that harbor a leading and a trailing process oriented horizontally, thus exhibiting a T-shape. ${ }^{14), 15)}$ After a stationary period, the granule cells switched to radial migration in the direction of the descending processes. The switching of migration mode from tangential to radial was also analyzed in detail in PN neurons by time-lapse analysis. ${ }^{16)}$ Here, two distinct types of switching were observed. When these neurons arrived at the presumptive $\mathrm{PN}$ region, the leading processes of some neurons began to deflect radially, which was followed by radial migration of the soma. In other PN neurons, new processes began to elongate in a radial direction from the soma of tangentially oriented neurons, following which the cells switched from tangential to radial migration. Thus, although certain types of neurons simply migrate radially to reach their final destinations, others reach their destinations by switching between modes of migration.

b) Changing the direction of migration. Switching the migration mode between radial and tangential causes drastic changes in the direction of migration, in which the leading process appears to play an important role. Migrating neurons traveling straight towards a specific direction elongate largely simple and unbranched leading processes. Typical examples are tangentially migrating cortical interneurons in the IZ/SVZ ${ }^{17)}$ (Higuchi and Murakami, unpublished data) and tangentially migrating PN neurons. ${ }^{18)}$ In contrast, when observed in explant culture or in living embryos, cortical interneurons migrating in the MZ, in particular those undergoing non-directed tangential migration, elicit multiple leading process branches in different directions from which they choose one to move in a new direction. ${ }^{13), 19)}$ Similar behaviors are observed for migrating neuronal precursors taken from the anterior SVZ (SVZa) in culture. $^{20)}$ These types of direction changes are distinct from those of growing axons, in that growing axons turn to new directions by directly turning their growing tips. ${ }^{21)}$

The most extreme case for changes in the direction of migration is the reversal of direction. In this case, a new leading process emerges from the tail of a migrating neuron, and the original leading process is retracted to begin migration in the opposite direction. Such behavior can be observed for cortical interneurons migrating in the $\mathrm{MZ}^{13)}$ and $\mathrm{PN}$ neurons migrating within the presumptive nuclear region. ${ }^{18)}$

\section{A long and complicated journey}

For most neurons, their migratory histories involve the deployment of more than one migratory mode. This phenomenon is particularly conspicuous for neurons that have to migrate over long distances to reach their final destinations. These neurons display switching between modes of migration in distinct phases of their journeys. In the following sections, we use a few examples, including cortical interneurons, hindbrain precerebellar neurons and cerebellar neurons, to illustrate how neurons migrate to and settle at their final destinations.

1) Tangential migration of cortical interneurons. Cortical interneurons mostly originate from the medial ganglionic eminence (MGE) and caudal ganglionic eminence (CGE) of the subpallium. ${ }^{22)}$ These neurons migrate tangentially and enter the pallium to spread into the entire cortex. When observed in coronal slices of GAD67-GFP mice, in which virtually all GABAergic interneurons express GFP ${ }^{23)}$ the leading cohort of GFP-expressing interneurons enters the lateral part of the cortex at an early stage of embryonic day (E)12.5. ${ }^{17)}$ Once in the cortex, the GFP-expressing interneurons form two streams, one in the IZ/SVZ and the other in the $\mathrm{MZ}^{17}$ ) (Fig. 2). Time-lapse analysis showed that IZ/SVZ interneurons in coronal slices indeed migrate towards the mediodorsal part of the cortex. ${ }^{17)}$ GFP-labeled interneurons in both of these streams spread lateromedially as development proceeded, suggesting the occurrence of medially-directed tangential migration.

a) Tangential migration of cortical interneurons in the IZ/SVZ. Interneurons in the IZ/SVZ extend largely unbranched leading processes that are oriented towards the midline in fixed embryos, ${ }^{24)}$ suggesting that they are migrating straight towards the midline. This observation was directly confirmed by time-lapse imaging in vivo (Higuchi and Murakami, unpublished data). We have labeled interneurons by in utero electroporation of tdTomato at E11.5 and analyzed them in both fixed and living embryos at E14.5. The cortex of this stage of development is relatively thin, making it advantageous for imaging cells at a depth of the cortex. Accordingly, we found that interneurons in the IZ/SVZ migrate straight towards the midline.

b) Tangential migration of cortical interneurons in the $M Z$. In fixed preparations of coronal slices, while GAD67-GFP labeled interneurons in the IZ/SVZ are oriented medially, those in the MZ are not. When observed in fixed flat mount preparations of the mice 


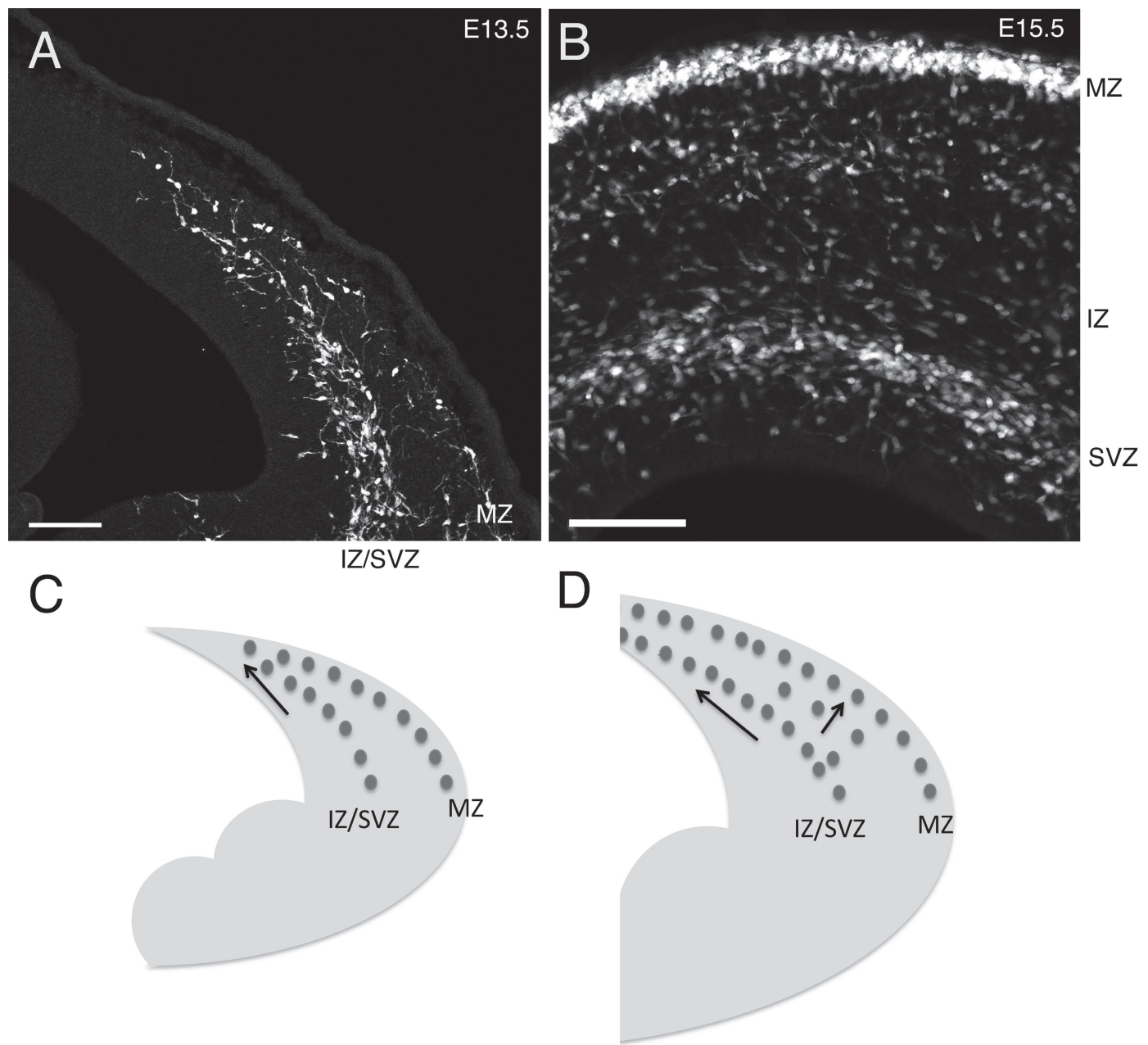

Fig. 2. The relationship between the two migratory streams of cortical interneurons. A, Coronal section showing the leading head of interneuron migratory streams in an E13.5 Nkx2.1 CreERT2; Ai9 cortex, in which tamoxifen was applied at E9.5. While the migratory streams in the MZ and IZ/SVZ are separated at the lateral part of the cortex, they appear to join at the medial part located at the leading head, a feature presumably reflecting the latero-medial sequence of cortical development (Kita et al., unpublished results). These neurons are likely the earliest generated interneurons, since the generation of interneurons begins at E10.5-E11.5 (Hevner et al., 2004). ${ }^{30)}$ Given that most CGE-derived interneurons are generated later than E13.5 and do not express Nkx2.1, these neurons must be mainly of MGE origin. B, A coronal section of an E15.5 GAD67-GFP mouse cortex. Migratory streams in the MZ and SVZ or IZ are segregated in their entire course. These migrating neurons should include both MGE and CGE-derived neurons. C and D, Schematics illustrating the migratory streams of interneurons in the cortex at E13.5 (C) and E15.5 (D). Medial is to the left. Scale bars, $100 \mu \mathrm{m}$.

cortex, leading processes of GAD67-GFP labeled cortical interneurons in the MZ are oriented in all directions. ${ }^{17)}$ Likewise, MZ interneurons labeled by in utero electroporation at E12.5 are oriented in all directions in flat mount preparations of E15.5 mice ${ }^{13)}$ (Fig. 3). In agreement with these observations in fixed embryos, live imaging using flat mount preparations showed that MZ interneurons migrated in all directions in the tangential plane. ${ }^{13), 17), 24)}$

This multidirectional migration was confirmed by in vivo imaging studies. The first attempt of imaging migrating neurons in living embryos was done by Yokota and colleagues ${ }^{25)}$ who used Dlx5/6Cre-IRES-EGFP mice to observe the behavior of MZ interneurons at E15. In their brief period of observation $(75 \mathrm{~min})$ they showed that these interneurons migrate in all directions. Recently, we carried out detailed observation of MZ interneuron migration in living mouse for a long-period of time $(>10 \mathrm{hr})$ in a stable condition. In this study we carried out quantitative analyses and confirmed that, at E16.5, these neurons also migrate in all directions ${ }^{19)}$ (Fig. 4). 

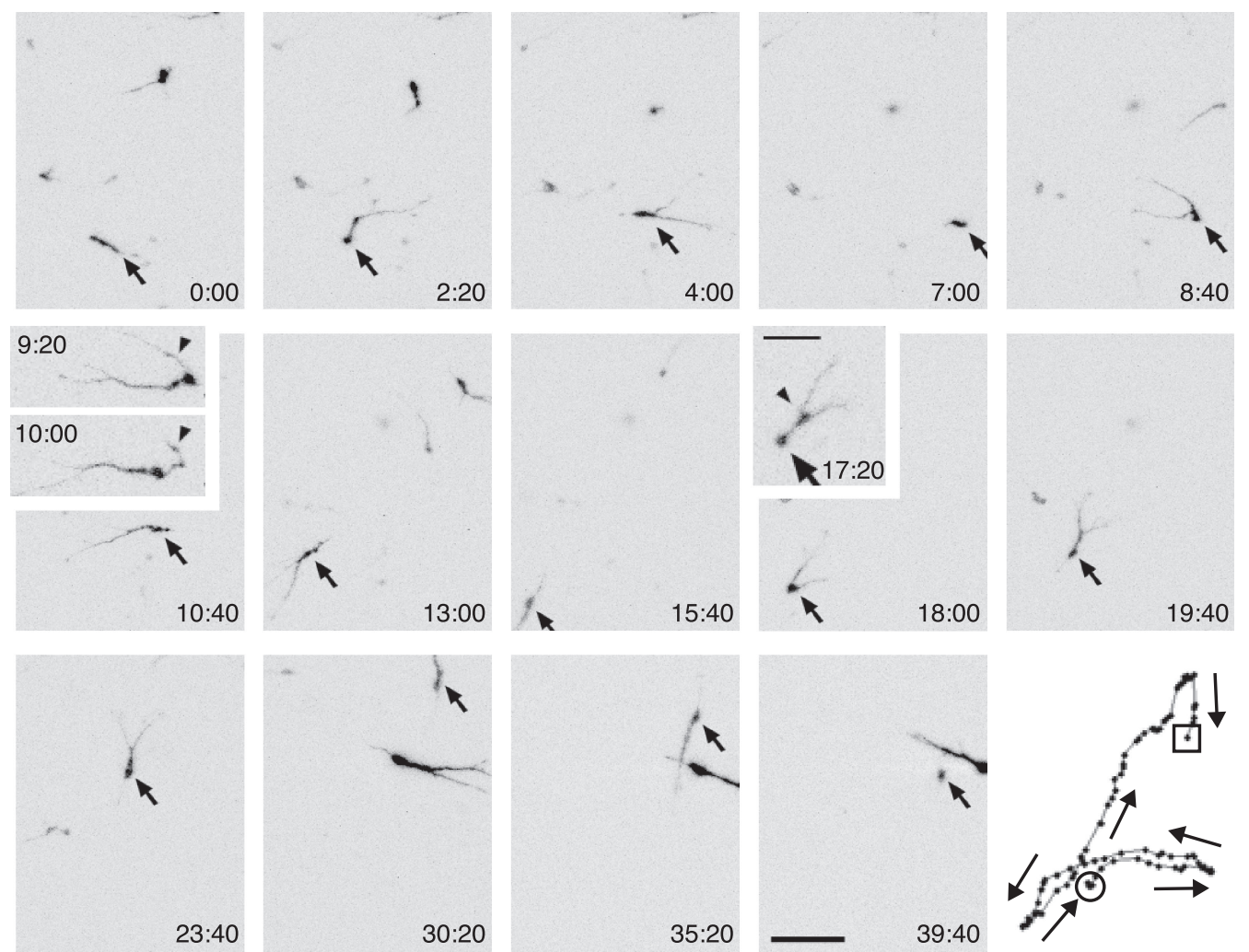

Fig. 3. Time-lapse sequence of a migrating interneuron in the MZ of a flat mount preparation of an E15.5 cortex visualized by electroporation of GFP at E12.5. ${ }^{13)}$ Arrows indicate the same neuron at different time points. In frames $9: 20$ and $10: 00$, the arrowheads indicate a leading process transforming into a trailing process. In frame $17: 20$, the arrow and arrowhead indicate a soma and a swelling, respectively. The numbers in the lower right or upper left corners indicate time. The panel in the lower right indicates tracking of the cell, with arrows pointing along the migratory directions. Scale bars, $50 \mu \mathrm{m}$ and $25 \mu \mathrm{m}$ (inset).

To conclude, these studies indicate that MZ cortical interneuron migrate in all directions in the tangential plane of the cortex.

c) Relationship between migrating interneurons in the IZ/SVZ and $M Z$. Observations in living embryos together with those in coronal slices indicate that medially-directed migratory behaviors are prominent for individually migrating interneurons in the IZ/ SVZ. In contrast, in either flat mount cortical preparations ${ }^{13), 17), 24)}$ or in vivo preparations, ${ }^{19)} \mathrm{MZ}$ interneurons do not show notable medially-directed migration. Instead, they migrate in all directions, ${ }^{19), 25), 26)}$ with many showing random walk behavior. ${ }^{13)}$

However, MZ interneurons also spread medially as development proceeds. One possible explanation for this medially-directed spread is that MZ interneurons are supplied from the IZ/SVZ. Consistent with this idea, at the front of the migrating streams, the MZ and IZ/SVZ meet at the early stages of development (Fig. 2C). These observations raise the possibility that neurons that have migrated through the IZ/SVZ reach the MZ at their leading head. MZ interneurons supplied in this way may explain the apparent latero-medial spread of MZ interneurons, at least in part.

As development proceeds, the streams of interneurons in the IZ/SVZ and the MZ begin to separate along the mediolateral extent of the cortex (Fig. 2B, D). However, the supply of interneurons from the IZ/SVZ to the MZ continues, as evidenced by the observation that interneurons translocate from the IZ/SVZ to MZ at E15.5. ${ }^{17)}$ The translocating interneurons appear to switch their mode of migration from tangential to radial. Taken together with the finding that the zonal distribution of interneurons labeled at E12.5 changes from the IZ/SVZ to the MZ with development, ${ }^{13)}$ these findings raise the possibility that the occurrence of interneurons in the MZ is an outcome of the translocation of interneurons from the IZ/SVZ.

A similar translocation can be observed for interneurons derived from the CGE. These neurons 

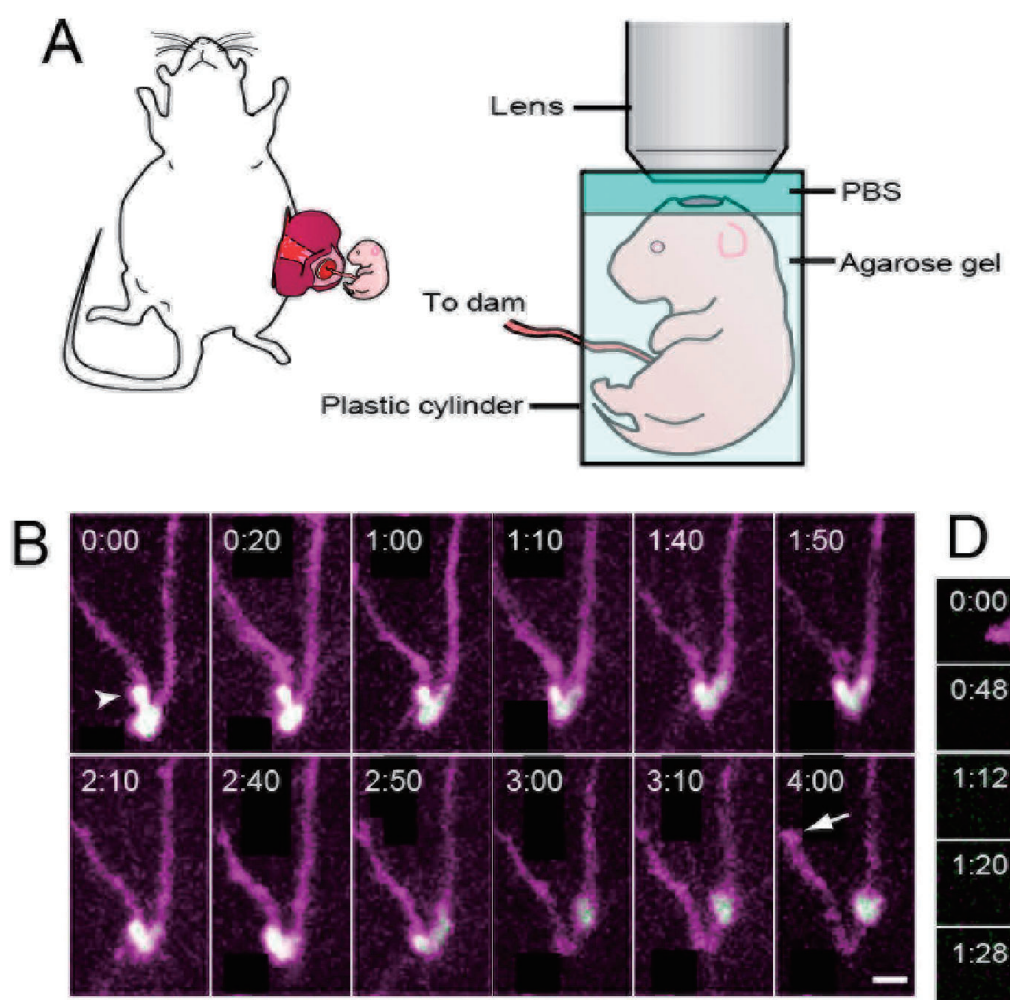

C
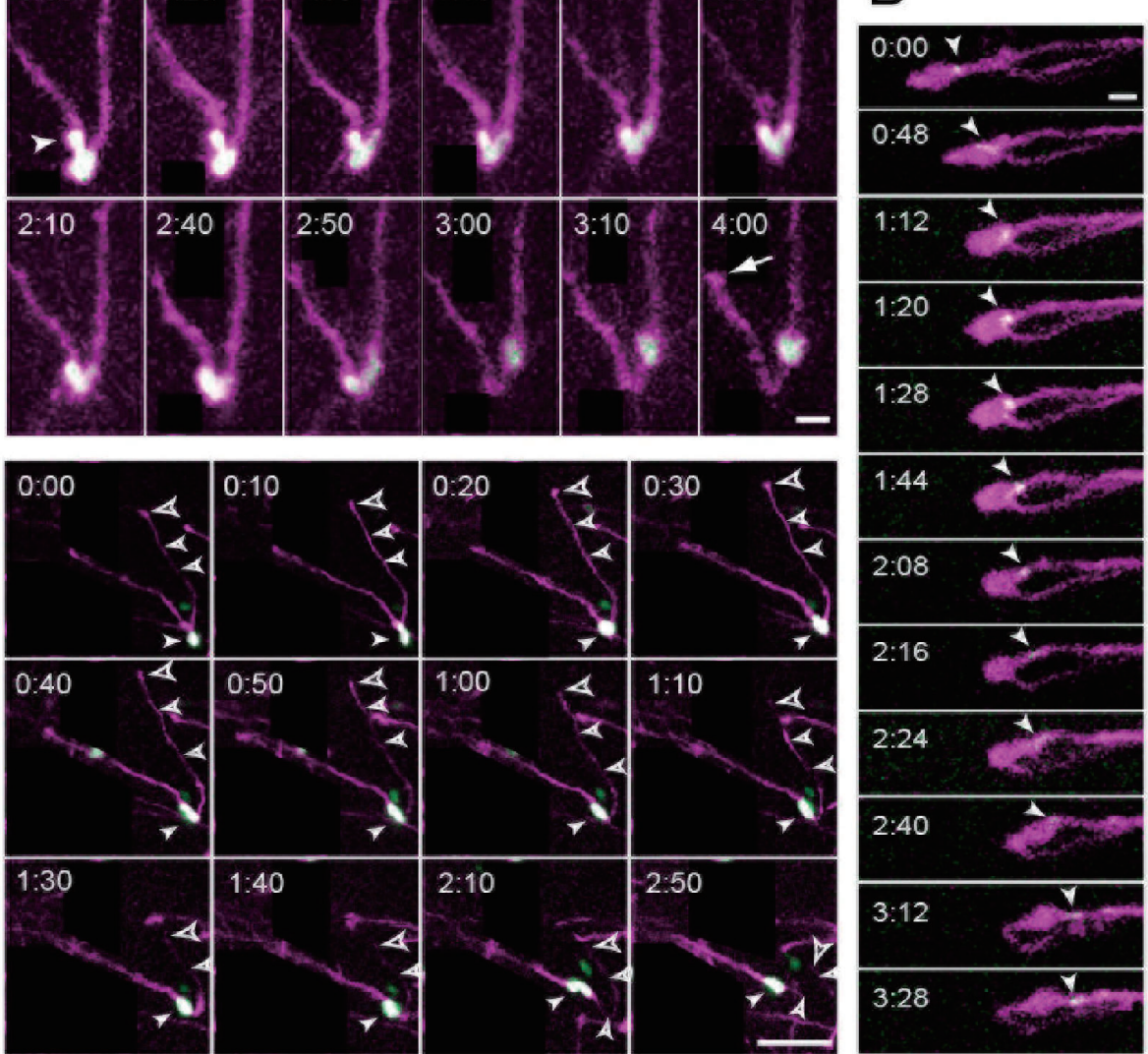

E

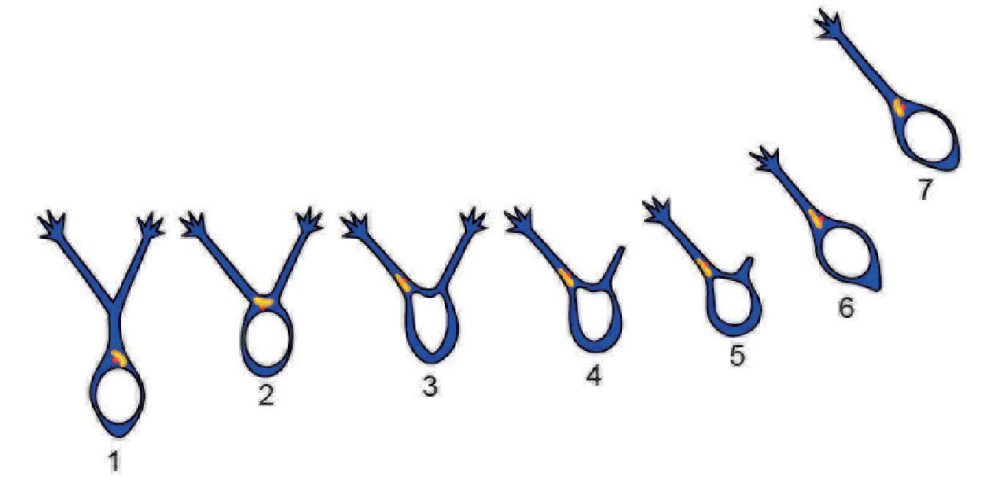

(Fig. 4) 
Fig. 4. Dynamics of the nucleus and Golgi apparatus at the bifurcating point of the leading process in living embryo. A, A schematic illustration of in vivo imaging in mouse embryos. See Yanagida et al., 2012 ${ }^{19)}$ for details. B, Time-lapse sequence showing the swinging behavior of a nucleus with $\mathrm{V}$-shaped morphology at a bifurcating point of the leading process. Neurons were labeled by electroporation of nuclear localization sequence (nls)-EGFP (green) and GAP-tdTomato (magenta). Arrowhead points to a budding form of the nucleus. The arrow points to the tip of the retracting process. Processes originating from cells that were excluded from this analysis are covered by black rectangles. C, Time-lapse sequence showing nucleokinesis near a branching point. Neurons were labeled by the electroporation of nls-EGFP (green) and GAP-tdTomato (magenta). The filled arrowheads indicate the nucleus, while the open arrowheads point to the branch that will eventually retract, with the larger arrowheads pointing to the tip of the branch. Two leading processes were extending when the nucleus reached the branching point $(t=0: 20)$. Subsequently, the nucleus directly entered one of the leading processes $(t=0: 30)$, followed by retraction of the other $(t=0: 50)$. Neurons that were excluded from the analysis are covered by black rectangles. D, Time-lapse sequence showing the dynamics of the Golgi apparatus at the bifurcating point of the leading process. The Golgi apparatus is shown as a white spot (arrowhead). Note that in most cases, the Golgi apparatus moved into a branch $(\mathrm{t}=1: 44)$ before the nucleus did. E, A model of the dynamics of subcellular organelles at the branching point of migrating cortical interneurons. At the branching point of the leading process (E2), the Golgi/centrosome complex moves into a leading process branch (E3) before the nucleus does (E4). Translocation of the Golgi/centrosome complex into a leading process appears to be a key step for selecting the new direction of migration. Elapsed time is indicated at the top-left corners (hr : min) in (B-D). Scale bars, $10 \mu \mathrm{m}(\mathrm{B}$ and D) and $50 \mu \mathrm{m}(\mathrm{C})$.

also enter the cortex, mostly by tangentially migrating through the IZ/SVZ ${ }^{27), 28)}$ and then radially migrate towards the CP and MZ. ${ }^{28), 29)}$

d) Termination of migration and final settlement in the CP. Most cortical interneurons eventually settle in the CP. Changes in the zonal distribution after labeling interneurons by in utero electroporation or BrdU administration indicate that cortical interneurons in the MZ migrate down into the depth of the $\mathrm{CP}$ postnatally, ${ }^{13), 30)}$ although it is not known whether they use radial fibers or some other cellular substrate for their migration to the CP. Although a major population of MGE-derived neurons appears to reach the $\mathrm{CP}$ via the $\mathrm{MZ}$, a subset of cortical interneurons originating from the CGE appears to settle in the CP without passing through the MZ. ${ }^{27), 29)}$

2) Migration of cerebellar neurons. a) Radial migration. The cerebellum is composed of excitatory and inhibitory neurons, as is the case in most other regions of the CNS. Among these, inhibitory neurons, Purkinje cells, Golgi cells, stellate cells and basket cells are generated at the $\mathrm{VZ}$ of the cerebellar primordium. ${ }^{31)}$ The Purkinje cells, which are the only output neurons from the cerebellar cortex and become postmitotic at E10.5-12.5 in mice, ${ }^{32), 33)}$ migrate radially towards the pial surface during embryonic stages $^{34)}$ (Fig. 5A). Precursors of stellate and basket cells are also generated at the $\mathrm{VZ}$ and migrate radially, but continue migration within the white matter ${ }^{35), 36)}$ and become postmitotic only postnatally. Thereafter, they migrate radially towards the top of the molecular layer and then migrate tangentially before settling at their final position. ${ }^{37)}$

b) Tangential migration. Three types of cerebellar neurons are known to undergo tangential migration at the early stages of development.
Granule cells, unipolar brush cells and cells of the (excitatory) cerebellar nuclei, all of which are excitatory neurons, are generated at the upper rhombic lip located at the dorsal edge of the cerebellar primordium. ${ }^{38), 39)}$ Of these, precursors of the granule cells and cerebellar nuclei cells, after leaving the upper rhombic lip, migrate tangentially through a subpial stream, ${ }^{9), 38)-40)}$ while unipolar brush cells appear to migrate through the white matter ${ }^{41)}$ before settling in the inner granule cell layer of the cerebellar cortex (Fig. 5A) (granule cells and unipolar brush cells) or the white matter (cerebellar nuclei cells). This mode of migration is important for the spread of granule cells and unipolar brush cells into the cerebellar cortex and for the formation of cerebellar nuclei.

c) Radial migration that follows tangential migration. Among these cerebellar neurons, granule cells, which become postmitotic postnatally, ${ }^{42)}$ begin to migrate radially away from the external granule layer (Fig. 5B). These cells migrate along the process of the Bergman glia, ${ }^{6)}$ a specialized astrocyte exclusively present in the cerebellar cortex. They then further migrate past the Purkinje cell layer to settle away from the pial surface. As a consequence, the internal granule layer is established beneath the Purkinje cell layer. ${ }^{9)}$

3) Migration of precerebellar neurons in the hindbrain. a) Tangential migration. The most prominent example of long distance tangential migration in the hindbrain comprises a subset of neurons that originate from the lower rhombic lip. After birth, these neurons form at least three migratory pathways, which lead to the formation of several discrete nuclei in the hindbrain. All of them relay neuronal information to the cerebellum and 
A

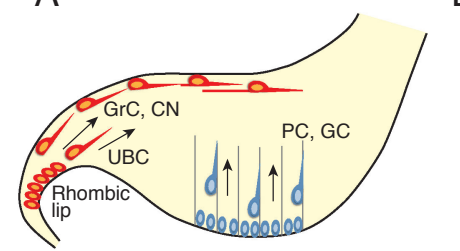

B

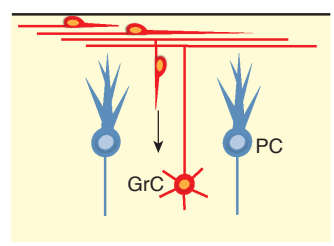

Fig. 5. Schematic illustration showing migration of cerebellar neurons. A, During embryonic stages Purkinje cells (PC) and Golgi cells (GC) originating from the cerebellar ventricular zone migrate radially towards the pial surface, while granule cell (GrC) precursors and deep cerebellar neurons (CN) originating from the upper rhombic lip migrate tangentially beneath the pial surface. Unipolar brush cells (UBC), which also originate from the upper rhombic lip, also migrate tangentially but through the white matter. B, During the postnatal development granule cells that initially migrate tangentially change the direction of migration and initiate radial migration. They migrate past the Purkinje cells to settle in the inner granule cell layer.

thus are named precerebellar nuclei. Among these, neurons that form the LRN and the external cuneatus nucleus (ECN) migrate circumferentially beneath the pia cross the ventral midline, forming the posterior extramural migratory stream $(\mathrm{PES})^{7), 10), 43)}$ (Fig. 6). Thereafter, they continue tangential migration to settle in positions away from the midline. A second pathway comprises neurons that form the PN and the reticulo-tegmental nuclei of the pons (RTN), which migrate tangentially also beneath the pia, first anteriorly and then ventrally towards the ventral midline, and form the anterior extramural migratory stream (AES). A majority of these neurons settle into nuclei without crossing the midline. ${ }^{7), 11)}$ The third migratory pathway is formed by neurons destined to become the inferior olivary nucleus (IO). These neurons migrate submarginally a distance from the pia towards the ventral midline and terminate migration without crossing the midline. ${ }^{44}$

b) Radial migration that follows tangential migration. When approaching their final destinations, some tangentially migrating AES and PES neurons exhibit an interesting switch of migratory mode from tangential to radial, with which these neurons leave the pial surface and position in the deeper end of their respective nuclei ${ }^{7), 16)}$ (Fig. $\left.6 \mathrm{~B}, \mathrm{C}\right)$. The phase of radial migration is seen to be in close association with the processes of radial glia) (see Fig. 9A, C). However, it remains unknown whether the submarginally migrating IO neurons also switch their mode of migration.

c) Termination of migration and formation of the $P N$. Migrating precerebellar neurons eventually terminate migration and form nuclei. Nuclei are not

\section{A}

B

Metencephalon

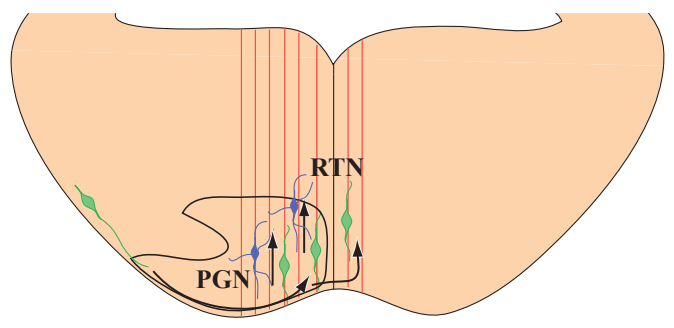

Myelencephalon

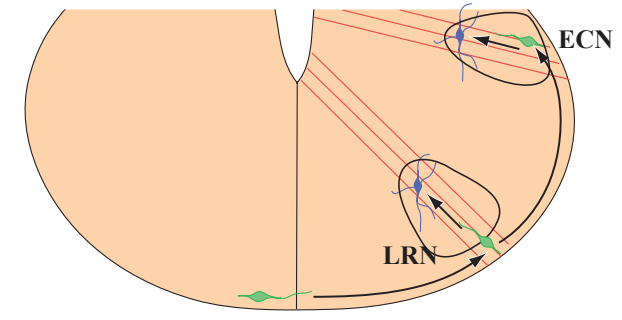

Fig. 6. Migratory routes of precerebellar neurons. Most precerebellar neurons originate from the lower rhombic lip (IRL) and migrate tangentially (A). Those that are fated to form the pontine grey nucleus (PGN) and the reticulo-tegmental nuclei of the pons (RTN) migrate through the anterior extramural stream (AES), while those that are fated to form the lateral reticular nucleus (LRN) and the external cuneatus nucleus (ECN) migrate along the posterior extramural stream (PES). The former migrate circumferentially beneath the pia matter and change the direction of migration from tangential to radial near the midline (B), mainly before crossing the ventral midline. The latter also migrate circumferentially and change the direction of migration from tangential to radial after crossing the midline (C). A is a lateral view, and B and $\mathrm{C}$ show cross sections of the hindbrain. Green cells represent migrating neurons and red lines represent fibers of radial glia.

uniform structures. For example, there is a precise topographic map for the efferents and afferents of the $\mathrm{PN},{ }^{45)}$ which suggests that termination of the migration of PN neurons should be a highly regulated process. The birthdate of PN neurons is correlated with their position within the $\mathrm{PN}$, with those born 
earlier positioned in its dorsal core and those born later forming the ventral outer shell. ${ }^{7), 11)}$ Interestingly, the migratory mode of early- and late-born PN neurons on their way to the presumptive $\mathrm{PN}$ region appears to differ. Early-born PN neurons tend to switch from tangential to radial migration, and hence settle in the deeper dorsal core of the PN. By contrast, late-born PN neurons migrate tangentially into the $\mathrm{PN}$ without switching radially, terminating at the ventral shell of the PN. ${ }^{18)}$ From this example, we can see that the dynamics of migratory modes that each subset of neurons adopts during the termination of migration may underpin the final positions within the nucleus.

Within the presumptive nuclear region, some PN neurons also display another type of dynamic behavior in that they reverse their originally mediallydirected tangential migration to laterally-directed migration. ${ }^{18)}$ This migratory strategy may be used to adjust the $\mathrm{PN}$ neurons' mediolateral positions within the PN. By contrast, PN neurons along the rostrocaudal dimension of the $\mathrm{PN}$ display very limited dynamics.

Precerebellar neurons, such as PN neurons, terminate their migration to aggregate into nuclei that have distinct three-dimensional shapes. This feature differs from the termination of migration by cortical interneurons and cerebellar granule cells, with the former terminating diffusely across the cortical plate and the latter into the internal granular layer. A three-dimensional view of how PN neurons terminate in the future nuclear region of the PN over the fourth dimension, time, as described above, suggests that the termination of migration is a dynamic process that involves neurons switching migratory modes or directions. Understanding how these dynamic and complex group activities are coordinated and regulated is a future challenge.

\section{Mechanisms that control the migration of neurons}

Migrating neurons must migrate forward, change their direction of migration, sometimes switch their mode of migration, and finally terminate their migration. In the following section we review the factors that control these processes.

a) Control of motility. Motility, which is essential for migration, can be regulated either by intrinsic or extrinsic factors.

i) Intrinsic motive force: Pulling and pushing forces on migrating neurons. Migrating neurons display distinct morphology, with a leading process in their

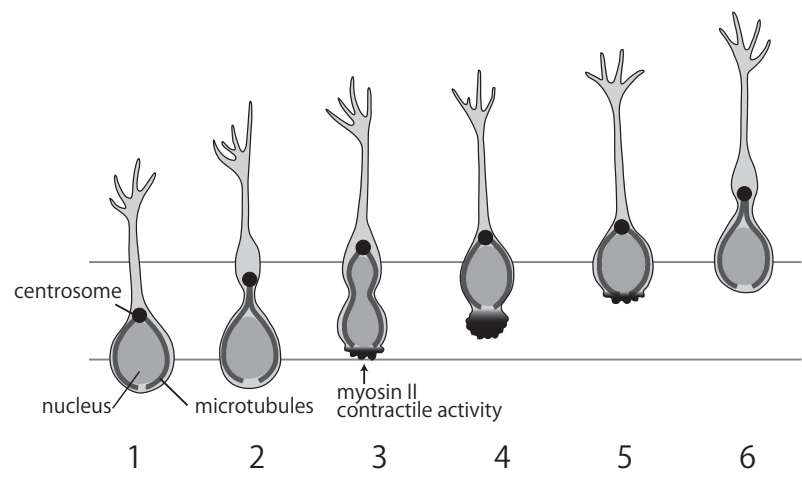

Fig. 7. A schematic illustrating the mode of two-step neuronal migration. The centrosome/Golgi complex (black dot) first moves ahead (1-3) and is followed by translocation of the nucleus (3-4). The nucleus is pushed forward by myosin, which is localized at the rear (3-5). Modified after Schaar and McConnell, 2005. ${ }^{50)}$

front, the cell soma, which is predominantly constituted of the nucleus, and a trailing process lagging in the rear. Interestingly, many migrating neurons display saltatory movement, in which extension of the leading process is followed by forward movement of the nucleus with a time lag. ${ }^{46)-49)}$ These morphological and behavioral characteristics have attracted attention to what drives the forward movement of the nucleus of a migrating neuron, a process termed nucleokinesis. Accumulating evidence suggests that pulling and pushing forces regulated by cytoskeletal proteins, such as microtubules and actin, and the actomyosin system play important roles in nucleokinesis.

It was previously proposed that nucleokinesis occurs in two steps, based on observations of neurons from the SVZa of rats on postnatal day (P) 0-2 cultured in Matrigel: first, forward movement of the centrosome, which is often associated with the Golgi apparatus, and second, forward movement of the nucleus (Fig. 7). ${ }^{50)}$ The forward movement of the centrosome, which is the main microtubule organizing center, pulls forward the longitudinal array of microtubules. The consequential absence of microtubules at the cell rear may trigger contractions mediated by myosin II, which generates a pushing force on the nucleus and serves to break adhesions at the cell rear. The role of actomyosin contraction at the rear also appears to be important for the migration of cortical interneurons. ${ }^{49)}, 51$ )

Migration also depends on pulling forces from the leading process. During the glia-supported migration of cerebellar granule cells, the majority of 
F-actin and myosin II is located at the front of the nucleus rather than at the trailing end, suggesting these proteins may pull the soma forward. ${ }^{52)}$ This idea was tested by $\mathrm{He}$ et al., who manipulated cytoskeleton dynamics and myosin II activity at restricted regions of migrating cerebellar granule neurons in dissociated culture by local perfusion with pharmacological agents. ${ }^{53)}$ They obtained evidence that a growth cone-like structure at the leading tip may pull the soma forward during neuronal migration through a myosin II-dependent forward flow of F-actin along the leading process. ${ }^{53)}$ However, both pulling and pushing mechanisms appear to work in tandem in the axophilic migration of Gonadotropinreleasing hormone (GnRH)-expressing neurons. ${ }^{54}$ )

ii) The role of motogen. Several extrinsic factors have been shown to accelerate migration. The best known is hepatocyte growth factor/scatter factor (HGF/SF). In mice lacking the urokinase-type plasminogen activator receptor, a key component of $\mathrm{HGF} / \mathrm{SF}$ activation, interneurons exhibit abnormal migration from the ganglionic eminence, which results in reduced interneurons in the frontal and parietal cortex, and exogenous application of HGF/ SF disrupts normal migration. ${ }^{55)}$ GABA, which may be secreted by the interneurons themselves, also appears to act as a motogen. Several lines of in vitro evidence suggest that GABA accelerates the tangential migration of cortical interneurons. ${ }^{56)-58)}$ This idea is supported by the in vivo imaging experiments done by Inada and colleagues, ${ }^{59)}$ who showed that application of a $\mathrm{GABA}_{\mathrm{A}}$ receptor antagonist to the surface of living postnatal mouse brain caused a decrease in the rate of migration of interneurons. Consistently, in GAD67-GFP mice, in which GABA content is reduced, the migration rate of interneurons was slower than that in vesicular GABA/glycine transporter (VGAT)-Venus mice, in which GABA content was unaffected. ${ }^{59)}$ These findings together suggest that extracellular GABA act as a motogen for cortical interneurons in neonatal mice.

b) Factors that control the route of migration. i) Radial migration. Cortical excitatory neurons migrating radially are thought to use the processes of radial glia as their scaffold. Indeed, electron microscopic observations showed that migrating excitatory neurons contact radial fibers in the neocortex. ${ }^{6)}$ In the cerebellum, during embryonic development, Purkinje cells, the principal neurons of and the only output neuron from the cerebellar cortex, migrate radially from the VZ towards the pial surface along the radial glia. ${ }^{34)}$
However, the radial glia is not the only group of cells used as scaffold for radial migration, as it is not present during the postnatal stage when cerebellar granule cells radially migrate down from the external granule layer. Instead, the processes of Bergman glial cells, a special type of astrocyte, ${ }^{60}$ ) are oriented perpendicularly to the pial surface and serve as the scaffold. ${ }^{6)}$

ii) Tangential migration. In contrast to radial migration, the scaffold for tangential migration has not been clearly defined. Since tangential migration of neurons in many cases occurs underneath the pial surface, it is possible that the pial meninges has a role. ${ }^{13), 61)}$ However, in some neurons, such as cortical interneurons that migrate through the IZ/SVZ ${ }^{17)}$ and IO neurons that migrate submarginally, no specific physically defined scaffold has yet been found.

While the physical substrates for tangentially migrating neurons remain ill-defined, it appears that localized expression of molecules may play a role in defining stereotypic migratory routes. Cortical interneurons migrate through two distinct routes, as described above. One factor contributing to this pathway selection is the localized expression of a chemokine, stromal derived factor SDF-1 (also known as CXCL12). SDF-1 is expressed not only in the pial meninges but also in the IZ/SVZ of the developing cortex, while its receptor, CXCR4, is expressed by interneurons migrating in the cortex. ${ }^{13), 62)-65)}$ SDF-1 can attract cortical interneurons both in vitro and in vivo. ${ }^{13), 62)}$ In CXCR4 or SDF1 knockout mice, cortical interneurons fail to follow the stereotypic MZ and IZ/SVZ pathways, but rather invade the dorsal telencephalon through the CP. ${ }^{13), 62), 65)}$ Thus, expression of SDF-1 along specific pathways seems to be important for the migration of interneurons along specific pathways.

A similar role for SDF-1 in confining migrating neurons to specific routes has been demonstrated in migrating precerebellar neurons in the hindbrain. SDF-1 is expressed in the pial meninges of the hindbrain, and CXCR4 is expressed in migrating precerebellar neurons. Precerebellar neurons are attracted towards a source of SDF-1 in vitro, and the marginal migratory routes of these cells are displaced to submarginal positions in either SDF-1 or CXCR4 knockout mice. ${ }^{66)}$

Repulsive molecules have also been implicated in determining the route of migration. MGE-derived interneurons migrate towards the cortex without invading the lateral ganglionic eminence (LGE). This phenomenon has been ascribed to two repulsive 
molecules, semaphorin $3 \mathrm{~A}$ and $3 \mathrm{~F}$, which are expressed in the LGE. ${ }^{67)}$ Their receptors, neuropilin-1 and -2 , are expressed in migrating interneurons. Consistently, cortical interneurons invade the LGE in neuropilin-2 knockout mice.

As can be seen from these examples, localized expression of attractive as well as repulsive molecules play a role in controlling the pathway of tangentially migrating neurons.

c) Factors that control the direction of migration. Most migrating neurons move towards specific directions. In theory, neurons can move in specific directions without external directional cues. Since migrating neurons are polarized and can move using scaffolds, such as the radial glia, a combination of these factors could explain their radial movement, analogous to trains moving in specific directions.

However, axon guidance molecules regulate the behaviors of migrating neurons in vitro. When SVZa cells are confronted with a gradient of the repellent molecule Slit, they reverse their direction of migration. This reversal is achieved either by new process formation or sequential formation of new branches in directions away from the source of Slit. ${ }^{20)}$ Axon guidance molecules have been shown to act as attractive cues for radially migrating cortical neurons. Semaphorin 3A, which is expressed in descending gradients across cortical layers, was shown to serve as an attractant for newborn cortical neurons, guiding them to the upper cortical layers. ${ }^{68)}$

There is evidence that a midline attractant is involved in the ventral midline-directed migration of PN neurons as well as that of LRN and ECN neurons in the hindbrain. In collagen gel culture, all these neurons are attracted by an explant of the midline tissue, and this attraction can be mimicked by COS cells expressing Netrin-1, ${ }^{69)-71)}$ a molecule widely known as an axon attracting molecule expressed in the ventral midline floor plate. ${ }^{72)}$ A receptor of Netrin-1, DCC, is expressed by PN neurons. In knockout mice of Netrin-1 and DCC, although PN neurons can leave their site of birth and migrate anteriorly, they fail to reach the midline. ${ }^{69)}$ Collectively, these lines of evidence indicate that PN neurons, and possibly LRN and ECN neurons as well, migrate towards the ventral midline in response to Netrin-1, which behaves as a midline attractant.

Intriguingly, changes in responsiveness to guidance cues also are important for migration. LRN and ECN neurons do not stay at the midline but continue tangential migration even after midline crossing. ${ }^{7), 43)}$ This is because they lose responsiveness to Netrin-1 upon encountering the floor plate. ${ }^{71)}$ Concomitantly, they acquire responsiveness to a yet unidentified alar plate-derived attractant. ${ }^{71}$

An attractant is also involved in the directed migration of MGE-derived interneurons towards the cortex. Neuregulin-1 (NRG1) is a member of the neuregulin family of proteins and contains an epidermal growth factor-like motif that activates membraneassociated tyrosine kinases related to the EGF receptor. Nrg1-Ig and Nrg1-CRD, a diffusible and a membrane-bound isoform of NRG1, respectively, are expressed in the developing cortex. $\left.{ }^{73}\right)$ On the other hand, the NRG1 receptor, ErbB4, is expressed in migrating interneurons. ${ }^{73), 74)}$ In vitro, Nrg1-Ig attracts MGE-derived cells, and these cells prefer Nrg1-CRD expressing substrate. In vivo loss of Neuregulin/ ErbB4 signaling causes a decrease in the number of cortical interneurons. These findings suggest NRG1 serves as a chemoattractant and also acts as a permissive molecule for MGE-derived interneurons.

d) Factors that regulate the termination of migration. Migrating neurons must terminate migration and settle when they arrive at their final destination. One possible mechanism for this termination is the existence of a factor ( $s$ ) in the extracellular milieu of the final destination that downregulates the motility. However, termination could also be regulated by intrinsic mechanisms.

We have studied termination using cortical interneurons as a model and found evidence for such intrinsic mechanisms. We carried out sequential in utero electroporation of GFP and mCherry to simultaneously label early-born and late-born interneurons, respectively, and compared their motility a few days after electroporation, when labeled neurons begin to execute tangential migration in the MZ, using cortical explants. ${ }^{75)}$ This experimental design allowed us to compare the motility of early- and late-born neurons in the same explant. We found that early-born neurons exhibited lower motility compared to late-born neurons. Since both of these neurons migrate under the same environment, this finding indicates that the motility of interneurons is intrinsically regulated. However, we also found evidence that the motility is regulated by a factor (s) secreted from cortical tissues. ${ }^{75}$ When E14.5 neurons were cultured on top of P7 cortical tissue, their motility was downregulated, which suggests that P7 cortical tissues secrete a factor (s) that reduces motility. ${ }^{75)}$ These findings together indicate cooperation between intrinsic and extrinsic factors when terminating interneuron motility. 
In general, however, little is known about the molecular mechanisms that terminate motility. A study of cerebellar granule cell migration revealed that the amplitude and frequency components of $\mathrm{Ca}^{2+}$ fluctuations are correlated positively with the rate of movement. ${ }^{76)} \mathrm{A}$ more recent study using cortical interneurons showed that the expression of the potassium-chloride co-transporter KCC2 leads to a reduction of the frequency of spontaneous intracellular calcium transients by reducing the membrane potential through $\mathrm{GABA}_{\mathrm{A}}$ receptor activation. ${ }^{77)}$ Thus, the developmental increase in the expression of $\mathrm{KCC} 2$, which would switch the GABA response of interneurons from depolarization to hyperpolarization, might reduce the amplitude and frequency components of $\mathrm{Ca}^{2+}$ fluctuations, leading to reduced motility. Consistent with this idea is that KCC2 immunoreactivity was substantially increased during early postnatal week and that early-born interneurons expressed higher levels of KCC2 compared to late-born interneurons. ${ }^{75)}$

e) Mechanisms underlying non-directed migration. In most cases, directed migration of neurons results in the formation of an aggregation of neurons such as the layers and nuclei. However, certain types of neurons do not form aggregates, but are dispersed in a wide area. Non-directed migration may be the mode of migration that contributes to this dispersion.

The most well described example of non-directed migration comes from cortical interneurons. Cortical interneurons initially migrate tangentially in a directed manner through the IZ/SVZ. Thereafter, they reach the $\mathrm{MZ}$, where they begin to migrate in all directions, sometimes changing their direction of migration as if they are wondering. ${ }^{13), 17), 19), 24) ~ A ~}$ theoretical analysis demonstrated that these interneurons in the MZ execute random walks. ${ }^{13)}$ Given that interneurons are distributed almost evenly in the mature cortex, this random-walk behavior may help interneurons spread throughout the cortex.

Like cortical interneurons, Cajal-Retzius cells also migrate tangentially near the pial surface. CajalRetzius cells are born in discrete loci of the pallium and migrate tangentially to colonize the entire cortex. $^{78), 79)}$ These neurons gradually spread into the entire cortex, during which they exhibit largely non-directed random-walk movement similar to cortical interneurons $^{80)}$ (Yanagida and Murakami, unpublished observation). Granule cells, stellate cells and basket cells of the cerebellum also migrate tangentially near the pial surface. Of these, stellate and basket cells appear to migrate in a non-directed manner ${ }^{37), 81)}$ (Hitoshi Komuro, personal communication), whereas granule cells migrate in a directed manner. ${ }^{82)}$ Although theoretical analysis is needed to confirm the random walk of stellate cells and basket cells, this migration pattern would likely contribute to the dispersion of these cells in the entire cerebellar cortex.

One possible mechanism underlying non-directed migration might be the lack of guidance cues or scaffold. Migrating neurons are directed towards or away from guidance cues, and in the presence of scaffold, migrating neurons move ahead along it. However, in the absence of such cues, persistence of cell polarity is weakened, which results in migrating neurons frequently changing their directions. In the case of Cajal-Retzius cells, there is evidence that contact repulsion between the cells, in addition to non-directed migration, also contributes to frequent changes in migration, thus leading to dispersion. ${ }^{80)}$

\section{Consequences of radial and tangential migration}

The occurrence of radial and tangential migration contributes to the creation of a heterogeneous neuronal milieu composed of varying types of neurons. In the neocortex, for example, while cortical progenitors exclusively generate excitatory neurons, inhibitory neurons immigrate from a distantly located structure in the subpallium by way of longdistance tangential migration, as described above. Likewise, in the cerebellar cortex, while VZ progenitors generate only inhibitory neurons, such as Purkinje cells and Golgi cells, ${ }^{31)}$ excitatory neurons such as granule cells and unipolar brush cells are generated in a neighboring germinal zone, the upper rhombic lip. While inhibitory neurons migrate radially, granule cells and unipolar brush cells tangentially migrate beneath the pial surface or through the white matter, respectively, and then radially migrate to settle in the depth (granule cell layer) of the cerebellar cortex, ${ }^{38), 39)}$ resulting in a mixture of excitatory and inhibitory neurons. Thus, a combination of radial migration and tangential migration, either directed or non-directed, generates a cytoarchitecture composed of both excitatory and inhibitory neurons despite the separate origins of these neurons. In the cerebellar and cerebral cortices, neuronal assembly is created as a consequence of the regulated migration of excitatory and inhibitory neurons from distinct progenitor origins, which lays down the basis for the functions of these structures (Fig. 8). 


\section{Consequences of neuronal migration: Transformation of the trailing process into the axon}

The termination of migration is not simply the end of a journey, but is the beginning of neuronal maturation. Mature neurons are polarized in a way that extends an axon on one side and several dendrites (mostly) on the other side of the cell. Migrating neurons are also polarized, but they extend a leading process in the front and a trailing process in the rear. A fundamental question that remains is how a migrating neuron that has a leading and a trailing process transforms into a mature neuron that has one axon and several dendrites.

Accumulating evidence suggests that the trailing process at the rear of a migrating cell turns into the axon in some neurons (Fig. 9A). A typical example is cerebellar granule cells. As described in the previous section, these neurons undergo tangential migration in the external granular layer and extend leading and trailing processes along the tangential axis. Then, they initiate radial migration along Bergman glial fibers, extending a radially oriented leading process in the front and a new trailing process at the rear. ${ }^{6), 14)}$ The trailing process becomes an axon, thus forming the T-shaped parallel fiber of these neurons. PN neurons in the hindbrain, may add yet another example. These neurons predominantly project axons to the contralateral cerebellum. They migrate circumferentially towards the ventral midline and extend a tangentially oriented leading process. Both observation of real time imaging and fixed samples showed that while a majority of migrating PN neurons settle at the vicinity of the midline without crossing it, their leading processes cross the ventral midline. ${ }^{7), 16), 18), 43)}$ Some PN neurons switch from tangential to radial migration as they settle, at which point they leave their original leading processes at the rear and migrate along the radial glia fibers with newly generated radially oriented leading process$\mathrm{es}^{7), 16), 43)}$ (Fig. 9B, C). While experimental confirmation is still awaited, it is likely that the original leading processes, which become the trailing processes as a consequence of $\mathrm{PN}$ neuron radial migration, may eventually turn into axons that project to the cerebellum. The trailing processes also appear to contribute to axons in cortical excitatory neurons, although this phenomenon shows a slight variation from the examples above. These neurons, soon after departing from the VZ, lose their neuroepithelial polarity and move to a non-polarized stage, which assumes a multipolar shape in the IZ/SVZ (the future white matter). ${ }^{5), 83)}$ There, they initiate axonal outgrowth tangentially. ${ }^{84-86)}$ Thus, in this case, axon formation occurs independently from the processes of the migrating cells. However, cortical excitatory neurons subsequently form radially oriented leading processes and begin radial migration into the cortical plate while they elongate their axons. ${ }^{84)}$ Since their trailing processes at the rear form a continuum with the axons, the trailing processes are thought to contribute to the radial part of the axons.

It should be noted that the axonal development of cortical interneurons is not related to the trailing process of migrating cells. These neurons display typical shapes of migrating neurons during embryonic development (see above). After a protracted period of multidirectional tangential migration, they descend from the MZ to the $\mathrm{CP}$ at perinatal stages. ${ }^{13), 30)}$ Yamasaki et al. (2010) performed long-time (>40 hr) live imaging experiments using organ culture preparations of perinatal stages, which recapitulate the in vivo development of cortical interneurons. ${ }^{87)}$ This study showed that cortical interneurons visualized by in utero electroporation at E12.5 in the MGE transiently assume a non-polarized sea urchin-like shape that alternately extends and retracts many short processes $^{87)}$ (Fig. 9D). Since these neurons do not show substantial changes in the position of their cell bodies, they may have terminated migration. At this point, one of the short processes suddenly elongates rapidly far beyond the length of the alternating extensions and retractions and acquires an axonal nature. These findings suggest that these neurons cease migration, which results in their morphological transformation from that of migrating neurons to a non-polarized sea urchin-like shape, and thereafter an axon develops de novo from the urchin-like cells.

It remains unknown what causes the difference in the mode of axon formation between cortical interneurons and other neurons. A common feature among cerebellar granule cells, a subset of PN neurons, and cortical excitatory neurons is that they migrate along the fibers of Bergman glia or radial glia at their final stages of migration during which their trailing processes might be associated with these fibers. These associations might stabilize the trailing process, enabling it to contribute to the axon. With regard to cortical interneurons, there is no evidence that they migrate along the radial glia when descending from the MZ to the CP. If they indeed descend independently of the radial glia, it may explain why these neurons are able to initiate axon 


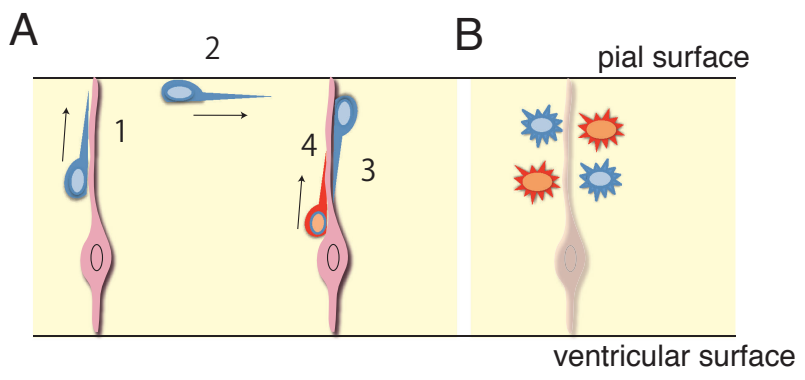

(Fig. 8)

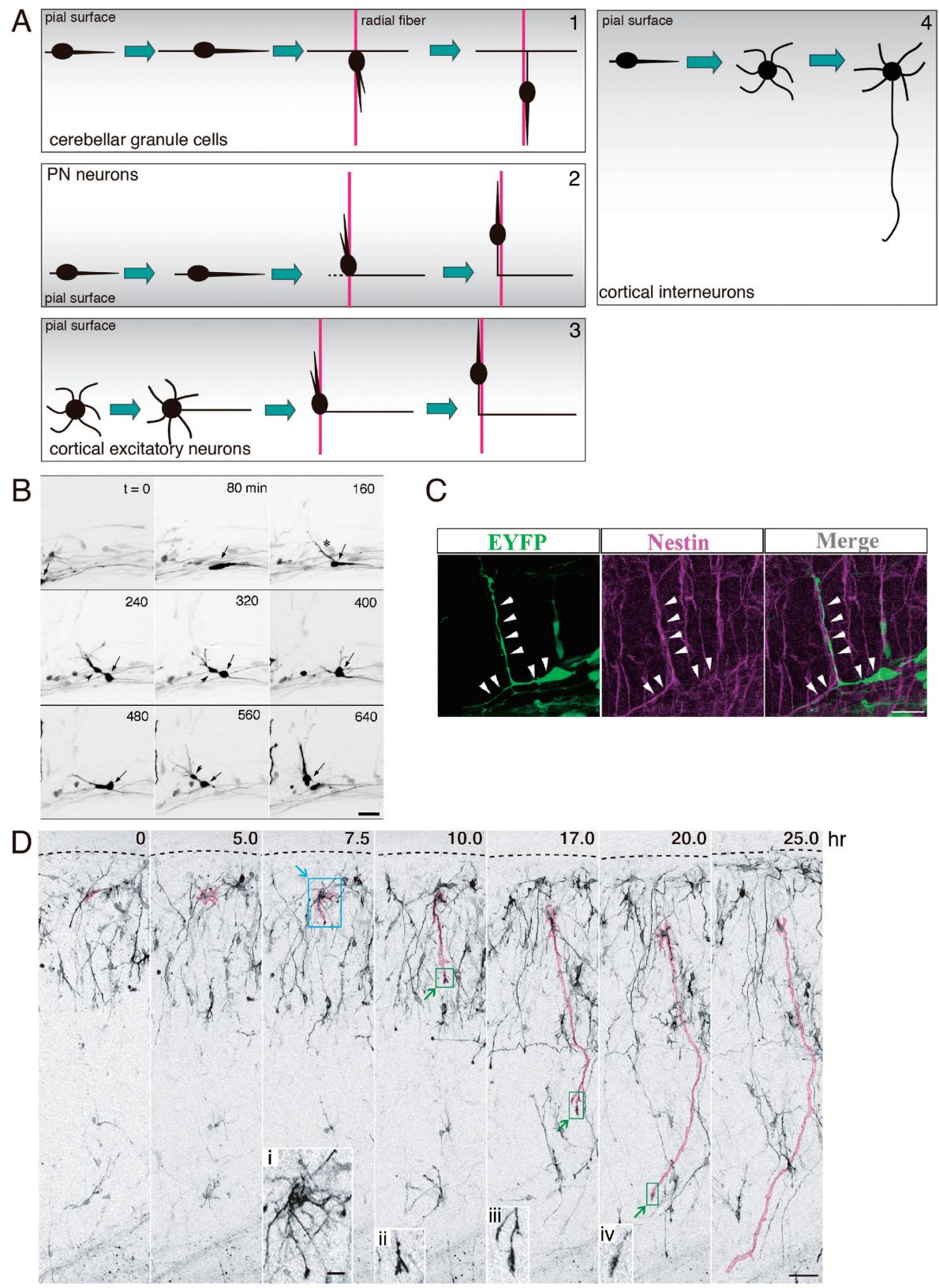

(Fig. 9) 
Fig. 8. A schematic illustration showing intermixing of neurons from different sites of origin as a result of switching the migratory mode. A, One neuron first migrates radially toward the pial surface (1), then tangentially (2), and finally radially back towards the ventricular surface (3) a distance away from the site of origin. Another neuron simply migrates towards the pial surface without much tangential displacement (4). B, Intermixing of different types of neurons as a result of tangential migration. Progenitors in different locations give rise to different types of neurons. Tangential migration of neurons, as shown in (A), allows neurons (blue) to move into a region where different types of neurons (red) are located.

Fig. 9. Axon elongation that follows neuronal migration. A, A schematic illustrating axon elongation for four types of neurons. A-1, Cerebellar granule cells first migrate tangentially through the external granule layer while extending a leading process in their front and a trailing process in their rear. Then, they switch their mode of migration from tangential to radial to migrate toward the internal granular layer along a radially aligned process of Bergman glial cells (red). The original leading and trailing processes that are left behind turn into the axon. A-2, PN neurons first migrate tangentially beneath the pial surface of the hindbrain while extending a leading process in their front. Upon arriving at the presumptive nuclear region, some PN neurons begin to migrate radially along the radial glial fiber (red). The original leading process, which has become the trailing process as a result of switching to radial migration, may turn into the axon. A-3, Cortical excitatory neurons exhibit a multipolar shape soon after departure from the ventricular zone. Then, they elongate a tangentially oriented process, which is followed by radial migration of the neuron along a radial glial fiber (red). The combined trailing process and tangential process turn into the axon. A-4, Cortical interneurons that have migrated tangentially in the MZ and descended into the CP assume a multipolar shape. The axon emerges from these non-polarized cells. B, Time-lapse sequence of the radial migration of a PN neuron (arrow). This neuron migrates tangentially from left to right. Upon arriving at the presumptive nuclear region, it slows down its migration and a new process (asterisk) emerges in a direction different from the direction of migration $(t=160)$, which induces radial migration of the cell $(t=640)$. Time in min is shown in the upper right corner of each panel. C, Extension of a radially oriented process from a PN neuron elongating along the radial fiber. Left panel shows EYFP-labeled PN neurons in the presumptive nuclear region; middle panel shows immnoreactivity of nestin, marker for radial glia cells; and right panel shows a merged view. D, Extension of an axon-like process from a sea urchin-like cortical interneuron. Time-lapse imaging began at P0.5. An axon-like process is marked in pink. Elapsed time after the onset of imaging (hr) is indicated on the upper right corners. Dashed lines indicate the pial surface. (i-iv) High magnification of the areas demarcated by blue or green rectangles show a sea urchin like-cell that has just initiated an axon-like process headed by a growth cone (i) and growing tips of axon-like processes (iiiv). Modified after Watanabe and Murakami, 2000, ${ }^{16)}$ Kawauchi et al. 20097) and Yamasaki et al., 2010. ${ }^{87)}$ Scale bars: $30 \mu \mathrm{m}$ (B and D), $20 \mu \mathrm{m}(\mathrm{C}), 10 \mu \mathrm{m}$ (inset in D).

formation in various directions that apparently have no relation to the direction of their trailing processes. Alternatively, it still remains possible that migrating cortical interneurons stop only transiently to initiate axons as excitatory neurons do and may resume migration thereafter resulting in trailing processes, which contribute to their axons. Further studies are necessary to validate this view. Nevertheless, the examples we describe here indicate that for certain types of neurons, processes during migration contribute to establishing axon-dendritic polarity.

\section{Concluding remarks}

Given that some neurons migrate long distances and settle in specific loci to establish neuronal connections, there is no doubt that proper regulation of neuronal migration is crucial for the establishment of the neuronal architecture. The mechanisms of migration have been studied using several systems, but most extensively using cortical interneurons, largely due to the functional importance of these neurons and their long-distance migration. Studies during the past two decades have unraveled details about the behavioral aspects and underlying mechanisms of cortical interneuron migration. However, it remains unknown to what extent these findings can be applied to other migrating neurons. For example, developmental upregulation of $\mathrm{KCC} 2$ was shown to play a critical role in the termination of interneuron migration. However, this may not be true for excitatory neurons such as cerebellar granule cells, Cajal-Retzius cells and hindbrain precerebellar neurons. While granule cells and PN neurons appear to use radial glia or Bergmann glial cells as scaffold for their radial migration away from the pia, whether cortical interneurons use the same strategy for their downward migration from the MZ remains unknown. Thus, an important question is whether there is yet unknown common mechanisms among the different types of neurons. Another fundamental but unclarified issue is how migrating neurons detect and transduce guidance cues in order to change their direction of migration. Although it is clear that the selection of the leading process branch is crucial, how migrating neurons detect concentration gradients and use this information is unknown. It is also important to know the common mechanism between the regulation of migrating neurons and growing axons. While these two dynamic events are distinct, there are several common properties, such as the 
neuron's response to attractive and repellent cues. Deepening our understanding of these issues is required to fully understand how the migration of neurons is regulated.

\section{Acknowledgements}

This work was supported by a Grant-in-Aid for Scientific Research (KAKENHI, 22220004 and 23123511) from the Ministry of Education, Culture, Sports, Science and Technology (Japan) to F.M. YH was supported by JSPS KAKENHI (25430020).

\section{References}

1) Levitt, P. and Rakic, P. (1980) Immunoperoxidase localization of glial fibrillary acidic protein in radial glial-cells and astrocytes of the developing rhesusmonkey brain. J. Comp. Neurol. 193, 815-840.

2) Götz, M. and Huttner, W.B. (2005) The cell biology of neurogenesis. Nat. Rev. Mol. Cell Biol. 6, 777788.

3) Brown, K.N., Chen, S., Han, Z., Lu, C.H., Tan, X., Zhang, X.J., Ding, L., Lopez-Cruz, A., Saur, D., Anderson, S.A., Huang, K. and Shi, S.H. (2011) Clonal production and organization of inhibitory interneurons in the neocortex. Science 334, 480486 .

4) Kriegstein, A.R. and Noctor, S.C. (2004) Patterns of neuronal migration in the embryonic cortex. Trends Neurosci. 27, 392-399.

5) Tabata, H. and Nakajima, K. (2003) Multipolar migration: the third mode of radial neuronal migration in the developing cerebral cortex. J. Neurosci. 23, 9996-10001.

6) Rakic, P. (1971) Neuron-glia relationship during granule cell migration in developing cerebellar cortex - Golgi and electronmicroscopic study in Macacus Rhesus. J. Comp. Neurol. 141, 283-312.

7) Kawauchi, D., Taniguchi, H., Watanabe, H., Saito, T. and Murakami, F. (2006) Direct visualization of nucleogenesis by precerebellar neurons: involvement of ventricle-directed, radial fibre-associated migration. Development 133, 1113-1123.

8) Parnavelas, J.G. (2000) The origin and migration of cortical neurones: new vistas. Trends Neurosci. 23. 126-131.

9) Komuro, H. and Yacubova, E. (2003) Recent advances in cerebellar granule cell migration. Cell. Mol. Life Sci. 60, 1084-1098.

10) Altman, J. and Bayer, S.A. (1987) Development of the precerebellar nuclei in the rat. 4 . The anterior precerebellar extramural migratory stream and the nucleus reticularis tegmenti pontis and the basal pontine gray. J. Comp. Neurol. 257, 529-552.

11) Altman, J. and Bayer, S.A. (1987) Development of the precerebellar nuclei in the rat. 3 . The posterior precerebellar extramural migratory stream and the lateral reticular and external cuneate nuclei. J. Comp. Neurol. 257, 513-528.
12) Chandrasekhar, A. (2004) Turning heads: development of vertebrate branchiomotor neurons. Dev. Dyn. 229, 143-161.

13) Tanaka, D.H., Yanagida, M., Zhu, Y., Mikami, S., Nagasawa, T., Miyazaki, J., Yanagawa, Y., Obata, K. and Murakami, F. (2009) Random walk behavior of migrating cortical interneurons in the marginal zone: Time-lapse analysis in flat-mount cortex. J. Neurosci. 29, 1300-1311.

14) Komuro, H., Yacubova, E., Yacubova, E. and Rakic, P. (2001) Mode and tempo of tangential cell migration in the cerebellar external granular layer. J. Neurosci. 21, 527-540.

15) Kawaji, K., Umeshima, H., Eiraku, M., Hirano, T. and Kengaku, M. (2004) Dual phases of migration of cerebellar granule cells guided by axonal and dendritic leading processes. Mol. Cell. Neurosci. 25, 228-240.

16) Watanabe, H. and Murakami, F. (2009) Real time analysis of pontine neurons during initial stages of nucleogenesis. Neurosci. Res. 64, 20-29.

17) Tanaka, D., Nakaya, Y., Yanagawa, Y., Obata, K. and Murakami, F. (2003) Multimodal tangential migration of neocortical GABAergic neurons independent of GPI-anchored proteins. Development 130, 5803-5813.

18) Shinohara, M., Zhu, Y. and Murakami, F. (2013) Four-dimensional analysis of nucleogenesis of the pontine nucleus in the hindbrain. J. Comp. Neurol. 521, 3340-3357.

19) Yanagida, M., Miyoshi, R., Toyokuni, R., Zhu, Y. and Murakami, F. (2012) Dynamics of the leading process, nucleus, and Golgi apparatus of migrating cortical interneurons in living mouse embryos. Proc. Natl. Acad. Sci. U.S.A. 109, 16737-16742.

20) Ward, M.E., Jiang, H. and Rao, Y. (2005) Regulated formation and selection of neuronal processes underlie directional guidance of neuronal migration. Mol. Cell. Neurosci. 30, 378-387.

21) Tessier-Lavigne, M. and Goodman, C.S. (1996) The molecular biology of axon guidance. Science 274, $1123-1133$.

22) Gelman, D.M. and Marín, O. (2010) Generation of interneuron diversity in the mouse cerebral cortex. Eur. J. Neurosci. 31, 2136-2141.

23) Tamamaki, N., Fujimori, K.E. and Takauji, R. (1997) Origin and route of tangentially migrating neurons in the developing neocortical intermediate zone. J. Neurosci. 17, 8313-8323.

24) Tanaka, D.H., Maekawa, K., Yanagawa, Y., Obata, K. and Murakami, F. (2006) Multidirectional and multizonal tangential migration of GABAergic interneurons in the developing cerebral cortex. Development 133, 2167-2176.

25) Yokota, Y., Gashghaei, H.T., Han, C., Watson, H., Campbell, K.J. and Anton, E.S. (2007) Radial glial dependent and independent dynamics of interneuronal migration in the developing cerebral cortex. PLoS ONE 2, e794.

26) Ang, E.S. Jr., Haydar, T.F., Gluncic, V. and Rakic, P. (2003) Four-dimensional migratory coordinates of GABAergic interneurons in the developing 
mouse cortex. J. Neurosci. 23, 5805-5815.

27) Miyoshi, G., Hjerling-Leffler, J., Karayannis, T., Sousa, V.H., Butt, S.J., Battiste, J., Johnson, J.E., Machold, R.P. and Fishell, G. (2010) Genetic fate mapping reveals that the caudal ganglionic eminence produces a large and diverse population of superficial cortical interneurons. J. Neurosci. 30, $1582-1594$.

28) Ma, T., Zhang, Q., Cai, Y., You, Y., Rubenstein, J.L. and Yang, Z. (2012) A subpopulation of dorsal lateral/caudal ganglionic eminence-derived neocortical interneurons expresses the transcription factor Sp8. Cereb. Cortex 22, 2120-2130.

29) Taniguchi, H., He, M., Wu, P., Kim, S., Paik, R., Sugino, K., Kvitsiani, D., Fu, Y., Lu, J., Lin, Y., Miyoshi, G., Shima, Y., Fishell, G., Nelson, S.B. and Huang, Z.J. (2011) A resource of Cre driver lines for genetic targeting of GABAergic neurons in cerebral cortex. Neuron 71, 995-1013.

30) Hevner, R.F., Daza, R.A.M., Englund, C., Kohtz, J. and Fink, A. (2004) Postnatal shifts of interneuron position in the neocortex of normal and reeler mice: Evidence for inward radial migration. Neuroscience 124, 605-618.

31) Hoshino, M., Nakamura, S., Mori, K., Kawauchi, T., Terao, M., Nishimura, Y.V., Fukuda, A., Fuse, T., Matsuo, N., Sone, M., Watanabe, M., Bito, H., Terashima, T., Wright, C.V.E., Kawaguchi, Y., Nakao, K. and Nabeshima, Y.I. (2005) Ptfla, a bHLH transcriptional gene, defines GABAergic neuronal fates in cerebellum. Neuron 47, 201-213.

32) Uzman, L.L. (1960) The histogenesis of the mouse cerebellum as studied by its tritiated thymidine uptake. J. Comp. Neurol. 114, 137-159.

33) Miale, I.L. and Sidman, R.L. (1961) An autoradiographic analysis of histogenesis in the mouse cerebellum. Exp. Neurol. 4, 277-296.

34) Yuasa, S., Kawamura, K., Kuwano, R. and Ono, K. (1996) Neuron-glia interrelations during migration of Purkinje cells in the mouse embryonic cerebellum. Int. J. Dev. Neurosci. 14, 429-438.

35) Zhang, L. and Goldman, J.E. (1996) Developmental fates and migratory pathways of dividing progenitors in the postnatal rat cerebellum. J. Comp. Neurol. 370, 536-550.

36) Yamanaka, H., Yanagawa, Y. and Obata, K. (2004) Development of stellate and basket cells and their apoptosis in mouse cerebellar cortex. Neurosci. Res. 50, 13-22.

37) Cameron, D.B., Kasai, K., Jiang, Y., Hu, T., Saeki, Y. and Komuro, H. (2009) Four distinct phases of basket/stellate cell migration after entering their final destination (the molecular layer) in the developing cerebellum. Dev. Biol. 332, 309-324.

38) Machold, R. and Fishell, G. (2005) Math1 is expressed in temporally discrete pools of cerebellar rhombic-lip neural progenitors. Neuron 48, 17-24.

39) Wang, V.Y., Rose, M.F. and Zoghbi, H.Y. (2005) Math1 expression redefines the rhombic lip derivatives and reveals novel lineages within the brainstem and cerebellum. Neuron 48, 31-43.

40) Fink, A.J., Englund, C., Daza, R.A., Pham, D., Lau,
C., Nivison, M., Kowalczyk, T. and Hevner, R.F. (2006) Development of the deep cerebellar nuclei: transcription factors and cell migration from the rhombic lip. J. Neurosci. 26, 3066-3076.

41) Englund, C., Kowalczyk, T., Daza, R.A., Dagan, A., Lau, C., Rose, M.F. and Hevner, R.F. (2006) Unipolar brush cells of the cerebellum are produced in the rhombic lip and migrate through developing white matter. J. Neurosci. 26, 9184-9195.

42) Altman, J. (1972) Postnatal-development of cerebellar cortex in rat. 1. External germinal layer and transitional molecular layer. J. Comp. Neurol. 145, 353-397.

43) Okada, T., Keino-Masu, K. and Masu, M. (2007) Migration and nucleogenesis of mouse precerebellar neurons visualized by in utero electroporation of a green fluorescent protein gene. Neurosci. Res. $\mathbf{5 7}, 40-49$.

44) Altman, J. and Bayer, S.A. (1987) Development of the precerebellar nuclei in the rat. 2 . The intramural olivary migratory stream and the neurogenetic organization of the inferior olive. J. Comp. Neurol. 257, 490-512.

45) Brodal, P. and Bjaalie, J.G. (1992) Organization of the pontine nuclei. Neurosci. Res. 13, 83-118.

46) Edmondson, J.C. and Hatten, M.E. (1987) Glialguided granule neuron migration in vitro: a highresolution time-lapse video microscopic study. J. Neurosci. 7, 1928-1934.

47) O'Rourke, N.A., Dailey, M.E., Smith, S.J. and McConnell, S.K. (1992) Diverse migratory pathways in the developing cerebral cortex. Science 258, 299-302.

48) Nadarajah, B., Brunstrom, J.E., Grutzendler, J., Wong, R.O.L. and Pearlman, A.L. (2001) Two modes of radial migration in early development of the cerebral cortex. Nat. Neurosci. 4, 143-150.

49) Bellion, A., Baudoin, J.P., Alvarez, C., Bornens, M. and Métin, C. (2005) Nucleokinesis in tangentially migrating neurons comprises two alternating phases: Forward migration of the Golgi/centrosome associated with centrosome splitting and myosin contraction at the rear. J. Neurosci. 25, $5691-5699$.

50) Schaar, B.T. and McConnell, S.K. (2005) Cytoskeletal coordination during neuronal migration. Proc. Natl. Acad. Sci. U.S.A. 102, 13652-13657.

51) Martini, F.J. and Valdeolmillos, M. (2010) Actomyosin contraction at the cell rear drives nuclear translocation in migrating cortical interneurons. J. Neurosci. 30, 8660-8670.

52) Solecki, D.J., Trivedi, N., Govek, E.E., Kerekes, R.A., Gleason, S.S. and Hatten, M.E. (2009) Myosin II motors and F-actin dynamics drive the coordinated movement of the centrosome and soma during CNS glial-guided neuronal migration. Neuron 63, 63-80.

53) He, M., Zhang, Z., Guan, C., Xia, D. and Yuan, X. (2010) Leading tip drives soma translocation via forward $\mathrm{F}$-actin flow during neuronal migration. J. Neurosci. 30, 10885-10898.

54) Hutchins, B.I., Klenke, U. and Wray, S. (2013) 
Calcium release-dependent actin flow in the leading process mediates axophilic migration. J. Neurosci. 33, 11361-11371.

55) Powell, E.M., Mars, W.M. and Levitt, P. (2001) Hepatocyte growth factor/scatter factor is a motogen for interneurons migrating from the ventral to dorsal telencephalon. Neuron 30, 79-89.

56) López-Bendito, G., Luján, R., Shigemoto, R., Ganter, P., Paulsen, O. and Molnár, Z. (2003) Blockade of $\mathrm{GABA}_{\mathrm{B}}$ receptors alters the tangential migration of cortical neurons. Cereb. Cortex 13, 932-942.

57) Heck, N., Kilb, W., Reiprich, P., Kubota, H., Furukawa, T., Fukuda, A. and Luhmann, H.J. (2007) GABA-A receptors regulate neocortical neuronal migration in vitro and in vivo. Cereb. Cortex 17, 138-148.

58) Cuzon, V.C., Yeh, P.W., Cheng, Q. and Yeh, H.H. (2006) Ambient GABA promotes cortical entry of tangentially migrating cells derived from the medial ganglionic eminence. Cereb. Cortex 16, 1377-1388.

59) Inada, H., Watanabe, M., Uchida, T., Ishibashi, H., Wake, H., Nemoto, T., Yanagawa, Y., Fukuda, A. and Nabekura, J. (2011) GABA regulates the multidirectional tangential migration of GABAergic interneurons in living neonatal mice. PLoS ONE 6 , $\mathrm{e} 27048$.

60) Buffo, A. and Rossi, F. (2013) Origin, lineage and function of cerebellar glia. Prog. Neurobiol. 109 42-63.

61) Borrell, V. and Marín, O. (2006) Meninges control tangential migration of hem-derived CajalRetzius cells via CXCL12/CXCR4 signaling. Nat. Neurosci. 9, 1284-1293.

62) Stumm, R.K., Zhou, C., Ara, T., Lazarini, F., Dubois-Dalcq, M., Nagasawa, T., Höllt, V. and Schulz, S. (2003) CXCR4 regulates interneuron migration in the developing neocortex. J. Neurosci. 23, 5123-5130.

63) Stumm, R., Kolodziej, A., Schulz, S., Kohtz, J.D. and Höllt, V. (2007) Patterns of SDF-1alpha and SDF-1gamma mRNAs, migration pathways, and phenotypes of CXCR4-expressing neurons in the developing rat telencephalon. J. Comp. Neurol. 502, 382-399.

64) Tiveron, M.C., Rossel, M., Moepps, B., Zhang, Y.L., Seidenfaden, R., Favor, J., König, N. and Cremer, H. (2006) Molecular interaction between projection neuron precursors and invading interneurons via stromal-derived factor 1 (CXCL12)/CXCR4 signaling in the cortical subventricular zone/intermediate zone. J. Neurosci. 26, 13273-13278.

65) López-Bendito, G., Sánchez-Alcaniz, J.A., Pla, R., Borrell, V., Picö, E., Valdeolmillos, M. and Marín, O. (2008) Chemokine signaling controls intracortical migration and final distribution of GABAergic interneurons. J. Neurosci. 28, 1613-1624.

66) Zhu, Y., Matsumoto, T., Mikami, S., Nagasawa, T. and Murakami, F. (2009) SDF1/CXCR4 signalling regulates two distinct processes of precerebellar neuronal migration and its depletion leads to abnormal pontine nuclei formation. Development 136, 1919-1928.

67) Marín, O., Yaron, A., Bagri, A., Tessier-Lavigne, M. and Rubenstein, J.L. (2001) Sorting of striatal and cortical interneurons regulated by semaphorinneuropilin interactions. Science 293, 872-875.

68) Chen, G., Sima, J., Jin, M., Wang, K.Y., Xue, X.J., Zheng, W., Ding, Y.Q. and Yuan, X.B. (2008) Semaphorin-3A guides radial migration of cortical neurons during development. Nat. Neurosci. 11, $36-44$.

69) Yee, K.T., Simon, H.H., Tessier-Lavigne, M. and O'Leary, D.D.M. (1999) Extension of long leading processes and neuronal migration in the mammalian brain directed by the chemoattractant netrin1. Neuron 24, 607-622.

70) Alcántara, S., Ruiz, M., De Castro, F., Soriano, E. and Sotelo, C. (2000) Netrin 1 acts as an attractive or as a repulsive cue for distinct migrating neurons during the development of the cerebellar system. Development 127, 1359-1372.

71) Taniguchi, H., Tamada, A., Kennedy, T.E. and Murakami, F. (2002) Crossing the ventral midline causes neurons to change their response to floor plate and alar plate attractive cues during transmedian migration. Dev. Biol. 249, 321-332.

72) Kennedy, T.E., Serafini, T., de la Torre, J.R. and Tessier-Lavigne, M. (1994) Netrins are diffusible chemotropic factors for commissural axons in the embryonic spinal cord. Cell 78, 425-435.

73) Flames, N., Long, J.E., Garratt, A.N., Fischer, T.M., Gassmann, M., Birchmeier, C., Lai, C., Rubenstein, J.L. and Marín, O. (2004) Short- and long-range attraction of cortical GABAergic interneurons by neuregulin-1. Neuron 44, 251-261.

74) Yau, H.J., Wang, H.F., Lai, C. and Liu, F.C. (2003) Neural development of the neuregulin receptor ErbB4 in the cerebral cortex and the hippocampus: preferential expression by interneurons tangentially migrating from the ganglionic eminences. Cereb. Cortex 13, 252-264.

75) Inamura, N., Kimura, T., Tada, S., Kurahashi, T., Yanagida, M., Yanagawa, Y., Ikenaka, K. and Murakami, F. (2012) Intrinsic and extrinsic mechanisms control the termination of cortical interneuron migration. J. Neurosci. 32, 6032-6042.

76) Komuro, H. and Rakic, P. (1996) Intracellular $\mathrm{Ca}^{2+}$ fluctuations modulate the rate of neuronal migration. Neuron 17, 275-285.

77) Bortone, D. and Polleux, F. (2009) KCC2 expression promotes the termination of cortical interneuron migration in a voltage-sensitive calcium-dependent manner. Neuron 62, 53-71.

78) Bielle, F., Griveau, A., Narboux-Nême, N., Vigneau, S., Sigrist, M., Arber, S., Wassef, M. and Pierani, A. (2005) Multiple origins of Cajal-Retzius cells at the borders of the developing pallium. Nat. Neurosci. 8, 1002-1012.

79) Takiguchi-Hayashi, K., Sekiguchi, M., Ashigaki, S., Takamatsu, M., Hasegawa, H., Suzuki-Migishima, R., Yokoyama, M., Nakanishi, S. and Tanabe, Y. (2004) Generation of reelin-positive marginal zone 
cells from the caudomedial wall of telencephalic vesicles. J. Neurosci. 24, 2286-2295.

80) Villar-Cerviño, V., Molano-Mazón, M., Catchpole, T., Valdeolmillos, M., Henkemeyer, M., Martínez, L.M., Borrell, V. and Marín, O. (2013) Contact repulsion controls the dispersion and final distribution of Cajal-Retzius cells. Neuron 77, 457-471.

81) Liesi, P., Akinshola, E., Matsuba, K., Lange, K. and Morest, K. (2003) Cellular migration in the postnatal rat cerebellar cortex: confocal-infrared microscopy and the rapid Golgi method. J. Neurosci. Res. 72, 290-302.

82) Ryder, E.F. and Cepko, C.L. (1994) Migration patterns of clonally related granule cells and their progenitors in the developing chick cerebellum. Neuron 12, 1011-1028.

83) Noctor, S.C., Martínez-Cerdeño, V., Ivic, L. and Kriegstein, A.R. (2004) Cortical neurons arise in symmetric and asymmetric division zones and migrate through specific phases. Nat. Neurosci. 7, 136-144.

84) Hatanaka, Y. and Yamauchi, K. (2013) Excitatory cortical neurons with multipolar shape establish neuronal polarity by forming a tangentially oriented axon in the intermediate zone. Cereb. Cortex 23, 105-113.

85) Namba, T., Kibe, Y., Funahashi, Y., Nakamuta, S., Takano, T., Ueno, T., Shimada, A., Kozawa, S., Okamoto, M., Shimoda, Y., Oda, K., Wada, Y., Masuda, T., Sakakibara, A., Igarashi, M., Miyata, T., Faivre-Sarrailh, C., Takeuchi, K. and Kaibuchi, K. (2014) Pioneering axons regulate neuronal polarization in the developing cerebral cortex. Neuron 81, 814-829.

86) Sakakibara, A., Sato, T., Ando, R., Noguchi, N., Masaoka, M. and Miyata, T. (2014) Dynamics of centrosome translocation and microtubule organization in neocortical neurons during distinct modes of polarization. Cereb. Cortex 24, 13011310 .

87) Yamasaki, E., Tanaka, D.H., Yanagawa, Y. and Murakami, F. (2010) Cortical GABAergic interneurons transiently assume a sea urchin-like nonpolarized shape before axon initiation. J. Neurosci. 30, 15221-15227.

(Received Aug. 14, 2015; accepted Oct. 8, 2015)

\section{Profile}

Fujio Murakami was born in Aichi Prefecture in 1948 and graduated from Osaka University in 1971. He received Ph.D. degree in 1974 and worked as a research associate in Osaka University and then in the National Institute for Physiological Sciences. He became a Professor of the Graduate School of Engineering Science, Osaka University in 1988, and then a Professor of the Graduate School of Frontier Biosciences in 2002. Since 2009, he has been serving as a PREST Research Supervisor, Japan Science and Technology Agency. He discovered that new axon collateral and synapses are formed following partial denervation, when he was a Ph.D. student, in collaboration with Professor Nakaakira Tsukahara and Dr. Hans Hultborn. After he became independent, he studied the mechanisms of the axon guidance and discovered that responses to axonal

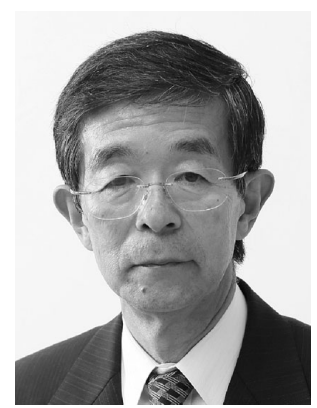
growth cones to guidance cues are not immutable, but can change by their encounter to guidance cues. This turned out to be a key mechanism for commissural axons to cross the midline and reach the contralateral side of the neural tube. He then moved into the field of neuronal migration. He directly visualized dynamics of migrating cortical interneurons both in vitro and in living embryos and discovered a novel mode of neuronal migration that violate previously thought significance of neuronal migration: interneurons in the cortical surface execute multidirectional migration modeled by a random walk. This mode of migration causes dispersal of interneurons into a wide area of the neocortex. 